\title{
Cyber Social Network Management: Enhancing the Learning Power of Distance Learners in Learning Communities
}

\author{
Zhong Zhixian and Shen Jianhua \\ Jiangxi Radio and TV University
}

\begin{abstract}
Based on the relevant concepts of Cyber Social Network Management (CSNM), this paper analyzes the necessity and possibility of CSNM from the perspective of connectivism and discusses the underlying aim and the essential strategies (processes, principles and tools) of CSNM. The paper makes an attempt to provide a series of helpful ideas and practices for distance learners to promote the learning power through CSNM.
\end{abstract}

Key words : Cyber Social Network Management, learning power, process, principle, tools.

\section{Concepts Delimitation: Cyberspace, Cyber Social Networks, Cyber Social Network Management \\ Cyberspace}

Cyberspace is the virtual space based on computer networks, which is opposite to the natural world. In an information/knowledge age, all of us live in two kinds of "worlds": one is the physical world which consists of atoms; the other is the digital virtual world, namely Cyberspace. Cyberspace is not a fantasy but a reality, because it possesses exponential shareable resources or wealth. Cyberspace is the virtual world filled with 'thinking' and information, and it can communicate in the fields of feelings, knowledge, ideas and culture using the information highway.

\section{Social Networks (SN)}

Social Networks (SN), namely social relationship networks, describe the relationships among people and organizations. Traditionally, a social network is an assemble of relationships among people in reality, while Cyber Social Networks (CSN) is the assemble of relationships among people and organizations in cyberspace, which is comprised nodes and links or connections.

\section{Cyber Social Network Management (CSNM)}

CSNM is the process and activity of creating, maintaining and using virtual social networks based on using social network tools and social software. 
A distance learning environment is open and virtual. Learners need large resources which are often stored in each node of their social network (a node refers to people, organizations or resource banks that are closely related to people) to solve problems. An effective Cyber Social Network depends onconscious and strategic management to form a connection which is targeted, steady and with an outstanding performance, for promoting an information/knowledge flow smoothly in a network. By the way of using various Social Network Tools (e.g., Social Networking Services such as Friendster, CiteUlike, Hotmail, Instant Message Systems, LotusNotes, Blog, Wiki, etc...) and creating, maintaining or constructing the social network that is in favor of learning, learners can obtain information, update knowledge, brainstorm and converge wisdom based on their learning needs.

\section{The Necessities and Possibilities of CSNM}

\section{Necessities}

* Adapting to the changing nature of learning metaphors. With the development of time, learning metaphors have been experiencing changes from behaviorism, cognitivism and constructivism to connectivism, i.e., from Reception (response-stimulus), Acquisition (assimilation-accommodation), Construction (meaning generation) to Connection (network forming/ network-creation). Connectivism is a learning theory that meets the need of the digital age, which is an important theoretical basis in network-based learning. It argues that learning is Network Forming (Siemens, 2004).

* Mastering new types of knowledge. In the digital age, one type of knowledge or learning content, i.e., "know where" and "know who" is becoming as important as or more important than "know-what", "know-why" and "know-how". Learning these types of knowledge ("know where" and "know who") depends on creating a rich and effective network, including the Social Networks (SN).

* Adapting to the change of learning culture. The learning culture has been changing in many fields. Firstly, many learners will move into a variety of different and unrelated learning fields in their life; secondly, informal learning is becoming an important learning experience in our education, and formal education (such as the traditional school education) is no longer the mainstay of our learning experience. Learning can happen in a variety of ways, such as communities of practice, personal networks and work-related tasks; thirdly, learning is a kind of continual process, lasting for a lifetime. As a whole, learning and work is the same thing, they are not separated as before. Finally, information technology (IT) is reconstructing the working mechanism of our mind (Siemens, 2004).

* Implementing distributed learning. Learning is not only an individual behavior, but a distributed group activity. With the help of a network formed by information technology, individual memory, cognition, intelligence, emotion and capability can be aggregated into a great learning power. As Karen Stephenson said, "Experience has long been considered 
the best teacher of knowledge. Since we cannot experience everything, other people's experiences, and hence other people, become the surrogate for knowledge. 'I store my knowledge in my friends' is an axiom for collecting knowledge through collecting people." (Siemens, 2004, para 14). Clearly, CSNM is an effective way to achieve distributed learning.

* Promoting collaborative learning. Essentially, learning is a social interaction. It depends on communication among all participants in learning. Effective communication based on Cyber Social Network (CSN) is one of the necessary conditions of distributed learning.

* Promoting the operation of the learning community. Learning community is a kind of learning approach or learning organization where learners complete their authentic tasks/problems by way of interdependence, exploration, communication and collaboration. The learning metaphor implied in a learning community is "learning is knowledge of social negotiation". A learning community includes four components: belongingness, sense of trust, reciprocities and sharing (Zhong, 2006, p. 199). An effective CSNM is the basis for operating an effective learning community..

Learning communities, knowledge construction communities and collaborative learning are of great value to distributed learning activities. They not only are in accord with the distributed nature of human cognition, but conducive to the development of learners' higher-order abilities, including analyzing, synthesizing, evaluating and creating. Effective distributed learning depends on learners to make full use of various tools to express their views/opinions. Therefore, the learner can achieve the purposes of communicating, sharing, debating, diverging views, aggregating wisdom of others in the community. The learner cannot form a community or communicate with each other without the CSN platform constructed by the learner.

\section{Possibilities}

* Six Degrees of Separation. It is a hypothesis of "Human Web" first created by Milgram in 1967 and demonstrated by Watts in 2002. It refers to the idea that everyone is at most, six steps away from any other person on Earth, so that a chain of "a friend of a friend" statements can be made to connect any two people in six steps or fewer. The "six steps" is just an approximate number, mainly used to explain that any two people can be linked in the world. Six Degrees of Separation not only explains the possibility of building Cyber Social Network Relationships (CSNR), but also reveals the widespread network of social or "weak ties" phenomenon. Learners can get any resource they need by way of using this kind of "weak ties".

* Age of Sharing. Digital age is a network era of sharing information resources. Sharing information is our nature. The technology-rich environment supports the possibilities of sharing. 
* Nature to Succeed. Managing CSN is a process of individual integration into a group, and it is a necessity for cultivating the nature to succeed. Psychologist Carl Jung, once constructed a well-known formula for success, i.e., "I + We = Fully I". The individual's power including knowledge, wisdom and emotions will generate a qualitative change when a person can be efficiently linked to a group. Group or team working is a prerequisite to succeed as a human being. Moreover, CSNM is an effective way of cultivating "Symphony", which is a comprehensive capacity of synthesizing diversities or multiple ideas and views of different fields. It is the ability to bring about diversities in harmony. The senses of symphony is one of the "six senses" emphasized in the book, A Whole New Mind: Moving From the Information Age to the Conceptual Age, by Daniel H. Pink. There are six essential aptitudes — "the six senses" - on which professional success and personal satisfaction increasingly will depend: Design, Story, Symphony, Empathy, Play and Meaning. These are fundamentally human abilities that everyone can master (Pink, 2005). The "six senses" or one of the six capabilities is a path and a result developing the right brain potentials and creative abilities.

* Social Networking Tools. With the arrival of Web 2.0, CSNM is becoming more convenient and fast. Learners can make use of a variety of interactive tools freely, such as synchronous and asynchronous interactive tools, and achieve various interactions effectively, such as autonomous, one-to-one, one-to-many, many-to-one and many-tomany based on Web 2.0 IT tools. Moreover, in support of CSN based on social software, learners can get support of academic, intelligence and interpersonal relationship in the network, and enlarge their learning power correspondingly.

\section{Enhancing Learning Power: The Essential Functions of CSNM}

\section{Learning Power}

Learning Power is sum of the learning motivation, perseverance, abilities, learning efficiency and transforming capabilities of learning. It can be explained as follows:

Learning Motivation: refers to the driving force of learning, including intrinsic motivation and external pressures.

Learning Perseverance: refers to the sustaining power of learning (someone who can persist in learning).

Learning Abilities: refers to the sum of the subjective and objective conditions of learning. Subjective condition means the abilities of receiving, assimilating and accommodating, constructing, generating and forming network in the process of learning. Objective condition refers to the material conditions needed in learning activities.

Learning Efficiency: it often means learning speed. 
Transforming Capabilities of Learning: refers to the transforming capabilities of knowledge, usually reflected in the effectiveness of self renewal, practice, creativity and social change.

\section{Learning Power: A Perspective of Connectivism}

It argues that the key theoretical base of CSNM is connectivism. In this perspective, the learning metaphor of adapting to the digital age is "Network-Forming". The learning power proposed and emphasized in connectivism is rich (Siemens, 2004, 2006). The function or objective of CSNM is mainly to promote the development of learning power needed in the digital age for the distance learner. (See Table 1).

Table 1: Learning Power: a Connectivist perspective

\begin{tabular}{|c|c|}
\hline $\begin{array}{c}\text { Learning } \\
\text { Power }\end{array}$ & Basic Content \\
\hline Connectivity & $\begin{array}{l}\text { The power of connecting specialized nodes or information sources; all } \\
\text { kinds of outside resources can be formed into an efficient, easily } \\
\text { retrieving knowledge network by the way of connecting "outside } \\
\text { brains" or "think-tanks", including specialized nodes, experts and } \\
\text { personal learning networks(PLN). }\end{array}$ \\
\hline Adaptability & $\begin{array}{l}\text { In a sense, intelligence is a kind of abilitiy in adapting to new } \\
\text { environments; learning to adapt to the workplaces of living, learning } \\
\text { and working; learning to adapt to cyber cultural environments. }\end{array}$ \\
\hline Sensitivity & $\begin{array}{l}\text { Keeping a clear-head, open, steady state to the time changes; keeping } \\
\text { the knowledge currency in order to update knowledge bank and } \\
\text { keeping pace with the times; changing one's mind with the situations } \\
\text { and making choices based on changes; learning to choose what to } \\
\text { learn, how to comprehend new information and make decisions in a } \\
\text { changing society. }\end{array}$ \\
\hline Searching & $\begin{array}{l}\text { The ability to "know more' is more critical; "know where" (the } \\
\text { understanding of where to find knowledge needed) and "know } \\
\text { who"(the understanding of who knows) is more important than } \\
\text { "know what", "know why" and "know how". }\end{array}$ \\
\hline Judgment & $\begin{array}{l}\text { The abilities of discrimination, evaluation and choice; identifying } \\
\text { whether it is important or not; evaluating whether it is true or false, } \\
\text { correct or wrong; choosing whether it is proper or not; decision- } \\
\text { making is based on choosing from schemes and information. }\end{array}$ \\
\hline Transference & $\begin{array}{l}\text { The abilities of transplanting knowledge and skills among fields, } \\
\text { mainly including the "Near Transfer" (e.g., "question-answer") and the } \\
\text { "Far Transfer"(e.g., "question-heuristic"). }\end{array}$ \\
\hline Practice & $\begin{array}{l}\text { Solving problems is the essence of practical power; it is actually the } \\
\text { process of problem-based solving or learning. The abilities "to do } \\
\text { something" may be in a practical sense (e.g., using a new software } \\
\text { tool) or refer to the abilities to enhance one's working performance in } \\
\text { knowledge society (e.g., self-awareness and personal information } \\
\text { management.). }\end{array}$ \\
\hline Creativity & Creativity means a kind of synthesizing abilities. It depends on seeking \\
\hline
\end{tabular}


and processing multiple views, having an insight into relationships among fields, ideas and concepts, combining the organizational learning and personal learning, and integrating academic knowledge with practical knowledge.

\section{Effective Performance: Strategies in executing CSNM}

\section{Process of CSNM}

CSNM refers to the processes and activities of constructing, regulating and making use of social network resources in the virtual space, including creating, maintaining connections and using social networks. They are not a direct and linear process but a cycle of "iterative and incremental" process, for example, using networks to maintain process, maintaining networks to utilize and create new connections in maintaining or utilizing process are common activities of CSNM.

\section{Creating Connections}

As other networks, CSN is formed by connections between two or more nodes. It comprises at least two elements: nodes and connections. These two factors directly determine "the size and richness" of CSN. The network will not keep on growing unless it increases nodes and connections continuously.

- Understanding the factors that affect the nodes to form connections.

Learning is the process of creating and forming networks in the perspective of connectivism. The key factors that affect or promote forming of connections are the following (Siemens, 2005).

* Motivation: Learners with clear objectives may have greater motivation to learn a new subject. Keller's (1987) ARCS model (Attention, Relevance, Confidence, Satisfaction) explains the possibilities of forming connections.

* Emotions: It plays an important role in evaluating valuable nodes and in tolerating the presence of contradictory perspectives. In addition, it greatly affects the activation of other nodes and is also used as a weighting coefficient in measuring elements in a network.

* Exposure: Repetition is a useful way of strengthening connections. A node grows in popularity or relevance and depends on linking more nodes.

$*$ Patterning: It is the process of recognizing the nature of and organizing various types of information and knowledge. It directly determines the degree of ease in forming new connections. For example, the obvious similarity between the patterns in two different academic fields will easily cause new connections and relevance: a learner in the field of medicine may recognize pattern similarity of elements between his/her field and the field of philosophy. 
* Logic: It provides certain occasions for nodes to form connections, and is one of the important factors to ensure the quality of new connections.

* Experience: It plays the role of catalyst in acquiring new nodes and forming connections in existing nodes.

- Locating, seeking and identifying nodes.

The nodes in CSN may include teachers, peers, experts and other individuals or organizations with common interests. These nodes relate to the learners' tasks directly or indirectly and facilitate the learner in accessing resources, diverging views, wisdom aggregating and problem-solving. The principle of centrality in network researches details the prominence of a node and the nature of its relation to the rest of the network. It provides us a lot of inspiration in creating meaningful connections (Siemens, 2005).

* Degree: Verifying the most influential nodes over the network.

* Closeness: Verifying the nodes with the quickest access to the highest number of other nodes within the network.

$*$ Betweenness: Verifying nodes that control the information flow of the network, sometimes referred to as "gatekeepers."

- Principle of minimum costs.

"Small World Effect" declares that most nodes within a network are connected by a fairly short path. Information flow from one domain of a well developed network to another generally requires a small number of "hops". We should learn to create a connection between nodes effectively by the way of a short path and less "energy".

\section{- Principle of Weak Ties.}

"Weak Ties" are links or bridges that allow short connections between information. The concept of weak ties expresses the understanding that much of our information comes from weak connections to networks other than our own. Our personal network is populated with nodes that are integrated with (or at least similar to) our own. For creativity or innovation, it often relies on unfamiliar, loose or weak connections to other less familiar networks. These connections provide a view into modes and manners of thought which are quite different from our own. Width of knowledge and thinking leads to variety and diversity, as a result of creativity or innovation. Creativity or innovation is often subject to familiarity or habit.

Nodes can form a specific interaction relationship by creating connections. Dialogue and collaboration are effective ways of creating connections. In the process of deep interaction between learners and alternative nodes, it marks the formation of connections between nodes with the amount of information flow among nodes. 


\section{Maintaining Connections}

Maintaining connections among nodes is key to a SN or CSN.

- Understanding basic characteristics of CSN

There are two basic characteristics for a CSN, one is self-organization and the other is adaptability.

Self-organization: A network is self-organized. A designer or instructor can influence the connections of new nodes, but the receptivity (and the nature of the existing learning network within a learner) will determine the integrating effectiveness of new information. Rocha (1998) defines self-organization as the spontaneous formation of well-organized structures, patterns, or behaviors. The injection of new nodes within a learning network can often be the instigating influence to a rapid reorganization. If the node significantly informs the existing structure, it can quickly become an illuminating hub.

Adaptability: A network is adaptive. The network will be regulated and change with the adjustment to the world. The nodes within the network will be continually updated to increase benefits to the network. It is verified in the field of human knowledge growth over the last half-century. The great advancements of science and society are greatly attributed to the increased capacities of connecting among people and organizations.

\section{- Mastering the influential factors in the knowledge flow.}

The factors that affect the knowledge flow can be known as follows. Firstly, "Flow Inhibitors" refers to the internal elements that reduce the possibilities of information/knowledge flow in a network, including biases, fixed ideas or inflexibility. Legitimate flow inhibitors can be internal inhibitors (cognition and emotion), such as some types of information that poorly fit in with the existing network or simply false information. It can also be external inhibitors (knowledge sharing culture in an environment) which impact the flow of information among learners and the degree of information flow among networks.

Secondly, "Flow Accelerators" refer to the internal elements and conditions that permit the formation of connections and distribution of information in a network. The elements that accelerate the formation of a network include receptivity, motivation, open culture, recognized value of collaboration, tools and time for collaboration.

\section{-Maintaining connections.}

* Interaction: It is the nature of networked learning. The types of interaction can be classified into four ways of interaction according to the number of nodes involved in an interaction, i.e., one-to-one, one-to-many, many-to-one and many-to-many. All ways of interaction can play roles in discussion about any topic and carry out collaborative tasks. 
Learners must learn to use a variety of social network tools effectively to support their social interaction in the virtual network. Different frequency of interaction determines whether the connection is weak or strong among nodes, and also determines the importance of each node in a learner's social network.

* Reflection: It includes reflection on whether the structure of a learner's social network is reasonable or not, whether the frequency and the ways of the interaction among nodes are appropriate or not, and the validity of social network tools.

* Initiative: It determines whether learners are willing to maintain connections with nodes, and willing to form a deeper connection with some nodes or not, and also determines the ways of maintaining and promoting the connections.

* Adjustment: Links between nodes are not unalterable. With the further interaction between learners and other nodes, further understanding with each other may occur. It requires learners to adjust the links appropriately in the process of maintaining connections.

\section{Using Connections}

Making use of human resources of a network to solve the relevant problem or to accomplish the task is the essential purpose of CSNM. In general, the purposes of utilizing the network resources have different learning objectives and relevant learning behaviors. (See Table 2).

Table 2: Objectives and behaviors in utilizing the network resources

\begin{tabular}{|l|l|}
\hline \multicolumn{1}{|c|}{ Objectives } & \multicolumn{1}{c|}{ Learning behaviors } \\
\hline $\begin{array}{l}\text { Information } \\
\text { sharing }\end{array}$ & $\begin{array}{l}\text { articulation, representation, connection, publishing, communication, } \\
\text { discussion, collaboration, reception, analysis, elaboration, integration, etc. }\end{array}$ \\
\hline $\begin{array}{l}\text { Information } \\
\text { collection }\end{array}$ & $\begin{array}{l}\text { recognition, selection, connection, communication, discussion, analysis, } \\
\text { question, identification, comparison, contrast, classification, generalization, } \\
\text { construction, integration, utilization, checking, evaluation, storage, } \\
\text { reflection, etc. }\end{array}$ \\
\hline $\begin{array}{l}\text { Knowledge } \\
\text { aggregation }\end{array}$ & $\begin{array}{l}\text { searching, selection, connection, discussion, communication, collaboration, } \\
\text { analysis, comparison, integration, construction, checking, confirmation, } \\
\text { evaluation, interpretation, reflection, storage, pattern-finding, transference, } \\
\text { etc. }\end{array}$ \\
\hline $\begin{array}{l}\text { Knowledge } \\
\text { construction }\end{array}$ & $\begin{array}{l}\text { interpretation, communication, debate, negotiation, collaboration, analysis, } \\
\text { comparison, Contrast, organization, integration, construction, generalization, } \\
\text { comprehension, elaboration processes, evaluation, utilization, reflection, etc. }\end{array}$ \\
\hline $\begin{array}{l}\text { Knowledge } \\
\text { correction }\end{array}$ & $\begin{array}{l}\text { collection, connection, communication, collaboration, understanding, } \\
\text { interpretation, compatibility, encouragement, analysis, inference, induction, } \\
\text { finding-support, checking, corroboration, discussing, operation, reflection, } \\
\text { etc. }\end{array}$ \\
\hline $\begin{array}{l}\text { Network } \\
\text { regulation }\end{array}$ & $\begin{array}{l}\text { analysis, communication, discussion, collaboration, evaluation, connection, } \\
\text { classification, reinforcing, reflection, etc. }\end{array}$ \\
\hline
\end{tabular}




\section{Basic principles of CSNM}

\section{Rule of 150}

It is one of the important sociological principles in social network. It states that the maximum number that can maintain a social interaction is 150 persons, i.e., the number of nodes in an effective interpersonal network should be within 150. Learners can get their resources by connecting the key nodes. Moreover, 150 persons in a network can communicate among themselves in a simple and short path; while preventing communication from weakening or falsifying in the process of information flow.

\section{The 80/20 Rule}

It is also known as the Pareto Principle (the law of the vital few, and the principle of factor scarcity). It states that, for many events, roughly $80 \%$ of the effects come from $20 \%$ of the causes. For example, " $80 \%$ of your sales come from $20 \%$ of your clients." or " $80 \%$ of the land was owned by $20 \%$ of the population". Based on this principle, learners should focus on managing $20 \%$ of the nodes in CSN, and interacting closely with these nodes (various information and knowledge flow within the nodes connections), for the reason that $80 \%$ resources for solving problems or accomplishing tasks may exist in $20 \%$ key nodes.

\section{Rule of Weak Ties}

The types of social relationships can be classified into weak ties and strong ties from the perspective of the frequency of interaction. In a social network with 150 nodes, 120 are weak ties, and 120 are strong ties. The rule of weak ties states that most of information we get is from the nodes and networks in weak ties. Creativity and innovation usually depends on utilizing unfamiliar, loose/weak ties. People can get more viewpoints and hints beyond thoughts from weak ties in a social network. Yet it must be noted that weak ties should be maintained, otherwise they will lose their original function or be broken away from a social network.

\section{IT Tools of CSNM}

The tools of CSNM mainly refer to the social networking tools or social software. The social networking tools are kinds of software of networking services, which can promote collaboration from bottom-up and creating/developing social networks for users. It makes the organization of information within the system and the social organization of users realize distant interaction synchronously in accordance with the social principles and mechanisms (Zhang, 2006). It is necessary to recognize and use the various interactions properly to master social networking tools in CSNM. The distant interactions include four types, i.e., one-to-one, one-to-many, many-to-one and many-to-many. Each type of interaction depends on relevant appropriate social networking tools. (See Table 3). 
Table 3: Four types of distant interaction and social networking tools

\begin{tabular}{|l|l|}
\hline \multicolumn{1}{|c|}{ Types } & \multicolumn{1}{|c|}{ Social network tools } \\
\hline $\begin{array}{l}\text { One-to- } \\
\text { one }\end{array}$ & $\begin{array}{l}\text { Mobile Phone, IP Telephone, Videophone, E-mail, Instant Message } \\
\text { Software (QQ, MSN, ICQ, UC, Trillian, etc.), Blog, Social Networking } \\
\text { Service (website, such as Friendster, Teachade, Google Group, etc.), FTP, } \\
\text { Social Bookmarking ...... }\end{array}$ \\
\hline $\begin{array}{l}\text { One-to- } \\
\text { many }\end{array}$ & $\begin{array}{l}\text { BBS, Wiki, Instant Message Software (QQ, MSN, ICQ, UC, Trillian, } \\
\text { etc.), Blog, Social Networking Service (Friendster, Teachade, Google } \\
\text { Group, etc.), Podcast, Social Bookmarking, Tag Clouds, Social } \\
\text { Networking Searching Engine, Web Syndication (RSS, etc.), Video } \\
\text { Conference..... }\end{array}$ \\
\hline $\begin{array}{l}\text { Many-to- } \\
\text { one }\end{array}$ & $\begin{array}{l}\text { Web Syndication (RSS, etc.), E-mail, Social Bookmarking, Blog, Video } \\
\text { Conference, FTP...... }\end{array}$ \\
\hline $\begin{array}{l}\text { Many-to- } \\
\text { many }\end{array}$ & $\begin{array}{l}\text { BBS, Social Bookmarking, USENET, Social Tagging, Online Video } \\
\text { Broadcasting, Web Syndication (RSS, etc.), Social Networking Service } \\
\text { (Friendster, Teachade, Google Group, etc.), Tag Clouds, Computer } \\
\text { Conferencing system, FTP...... }\end{array}$ \\
\hline
\end{tabular}

"One-to-one” social networking tool: It includes a variety of Instant Messaging Softwares (QQ, MSN, ICQ, UC, Trillian, etc.), E-mail, Blog, IP phone and a variety of Social Networking Services. These tools play a very important role in creating, maintaining and using CSN. For example, learners can publish their views about a certain topic in a Blog and other students can leave notes or send E-mails after reading the views to express their opinions. Moreover, they can have more frequent communication or dialogue through a variety of Instant Message Softwares.

"One-to-many" social networking tool: It includes a variety of Instant Messaging Softwares (QQ, MSN, ICQ, UC, Trillian, etc.), Social Networking Services, Blog, Wiki, Web Syndication (e.g., RSS) and BBS. These tools are mainly used in learners' interactions in a social network or other learning organizations.

"Many-to-one" social networking tool: It includes Web Syndication (e.g., RSS), Blog and E-mail. These tools are mainly used in the process of gathering information.

"Many-to-many" social networking tool: It includes a variety of Social Bookmarking, USENET, Social Networking Services, Tag Clouds and online video broadcasting. The learner can interact with another network as a node.

In brief, CSNM is an important part of self-management for distance learners. Networkbased distance learning in the digital age requires distance learners to get used to selfmanagement. Distance learners can enhance their learning powers, learning efficiency and effectiveness in communities effectively by the way of understanding relevant concepts and mastering useful strategies. 


\section{References}

Pink, D. H. (2005). A Whole New Mind [M]. USA: Penguin Group.

Rocha, Luis M. (1998). Selected Self-Organization and the Semiotics of Evolutionary Systems. In S. Salthe, G. Van de Vijver, \& M. Delpos (Eds.), Evolutionary Systems: The Biological and Epistemological Perspectives on Selection and Self- Organization (pp. 341-358). Norwell, USA: Kluwer Academic Publishers.

Siemens, G. (2004). Connectivism: A Learning Theory for the Digital Age. Retrieved from http://www.elearnspace.org/Articles/connectivism.htm on 10 October 2007.

Siemens, G. (2005). Connectivism: Learning as Network-Creation. Retrieved from http://www.elearnspace.org/Articles/networks.htm on 15 October 2007.

Siemens, G. (2006). Knowing Knowledge. Retrieved from http:www.elearnspace.org/KnowingKnowledge_LowRes.pdf on 23 October 2007.

Zhang, Shuren. (2006) Study from Social Software, Web2.0 to Complex Adaptive Information System (PhD thesis). Renmin University of China, Beijing, China.

Zhong, Zhi-xian. (2006). A Framework of Instructional Design Toward Knowledge Age: Promoting the Development of the Learner [M]. Beijing: China Social Sciences Press. 